\title{
LINE LOADS MOVING ON THE SURFACE OF AN INHOMOGENEOUS ELASTIC HALF SPACE
}

\author{
R. A. SCOTT \\ Dept. of Engineering Mechanics \\ The University of Michigan, \\ Ann Arbor (Mich.), U.S.A.
}

\begin{abstract}
Treated are line loads travelling with constant speed on the surface of an inhomogeneous elastic half space, the materials considered being such that uncoupled motions can arise. Assuming a quasi static state, solutions are presented for (i) a line force in the plane of the surface, acting in a direction perpendicular to that of its motion (called $\mathrm{SH}$ line load), and (ii) a normal line force moving in a direction perpendicular to its length.
\end{abstract}

\section{§ 1. Introduction}

Though many authors have treated elastodynamic problems involving inhomogeneous media (see, for instance, Vlaar [1], Lock [2], Karlsson and Hook [3]), so far, to the writer's knowledge, there has been no investigation of the interesting area of the response due to moving loads and allied topics such as crack propagation. As a first step in such a study, solutions are presented here to the problems of a concentrated SH load and a concentrated, normal, line load moving with constant velocity along the surface of an isotropic, inhomogeneous, elastic half space (see Fig. 1). In both cases it is assumed that an observer moving with the load sees a static pattern (quasi static assumption) and the analysis is restricted to certain classes of inhomogeneity for which the wave speeds are constant. For the $\mathrm{SH}$ load both subsonic supersonic velocities are treated, whereas only supersonic speeds and one type of inhomogeneity are analyzed for the normal line load. 

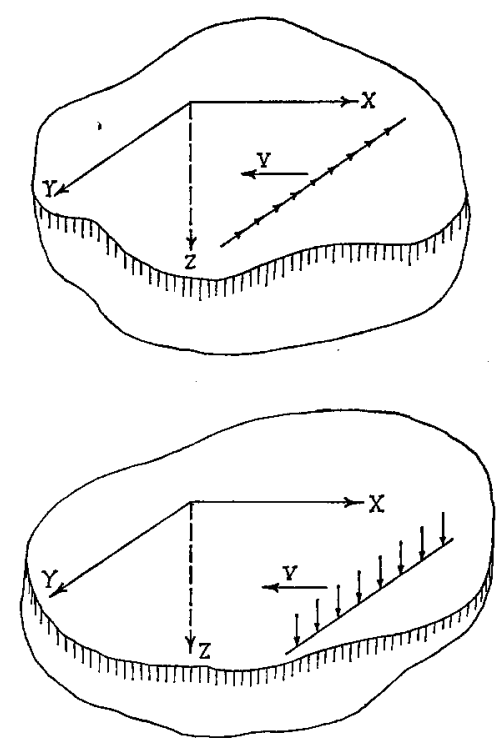

Fig. 1. Geometry of SH and normal line load problems. The top part represents the travelling SH line load, the lower part the travelling normal line load.

\section{§ 2. Analysis}

The displacement equation of motion for an inhomogeneous, isotropic, elastic solid is, in the absence of body forces (see Hook [4]),

$$
\begin{gathered}
\nabla[(\lambda+2 \mu) \nabla \cdot \boldsymbol{u}]-\nabla \times(\mu \nabla \times \boldsymbol{u})+2[(\nabla \mu \cdot \nabla) \boldsymbol{u}-(\nabla \mu)(\nabla \cdot \boldsymbol{u})+ \\
+(\nabla \mu) \times(\nabla \times \boldsymbol{u})]=\rho \frac{\partial^{2} \boldsymbol{u}}{\partial t^{2}},
\end{gathered}
$$

where $\boldsymbol{u}$ is the displacement vector, $\lambda$ and $\mu$ are the Lamé parameters, and $\rho$ is the density. With SH motions in mind, the following question is now posed in terms of Cartesian coordinates * $x, y, z$ : Can the assumptions that $u_{x}=0, u_{z}=0$, and all quantities are independent of $y$, lead to the satisfaction of (1) and to the vanishing of all stress components except $\sigma_{x y}$ and $\sigma_{y z}$ ? It is found that the answer is 'yes' provided $\mu$ is not a function of $y$ and the following

*) The $z$-axis is directed into the half space (surface: $z=$ constant) and in all cases the load is taken to be travelling along the negative $x$-axis. 
equation is satisfied

$$
\frac{\partial^{2} u}{\partial x^{2}}+\frac{\partial^{2} u}{\partial z^{2}}-a(x, z) u=\frac{\rho(x, y, z)}{\mu_{0} f(x, z)} \frac{\partial^{2} u}{\partial t^{2}},
$$

where $\mu_{0} f=\mu, \mu_{0}$ being a constant, and

$$
\begin{gathered}
u(x, z, t)=u_{y}(x, z, t) f^{\frac{1}{2}}(x, z) \\
a(x, z)=\frac{1}{4 f}\left[2 \frac{\partial^{2} f}{\partial x^{2}}+2 \frac{\partial^{2} f}{\partial z^{2}}-\frac{1}{f}\left(\frac{\partial f}{\partial z}\right)^{2}-\frac{1}{f}\left(\frac{\partial f}{\partial x}\right)^{2}\right] .
\end{gathered}
$$

The pertinent stress-displacement relation can be written

$$
\sigma_{y z}=\mu_{0} f^{2}\left(\frac{\partial u}{\partial z}-\frac{1}{2 f} \frac{\partial f}{\partial z} u\right)
$$

and the boundary condition is

$$
\sigma_{y z}=\sigma_{0} \delta(x+v t), z=0,
$$

where $\sigma_{0}$ and $v$ are constants and $\delta$ denotes the delta function.

The problem presented by eqs. (2), (4), and (5) is, in general, of considerable complexity. In order to obtain a tractable model, some simplifying assumptions are necessary. Henceforth, attention is restricted to media for which (i) $\mu$ and $\rho$ vary in the $z$-direction only. ${ }^{*}$ and they also have the same variation, so that the $\mathrm{SH}$ wave speed is constant, and (ii) the variation is such that $a$ as given by (3) is a constant. Listed below are several cases for which property (ii) holds

Case I $f(z)=$ constant $(=1), a=0$

Case II $f(z)=\exp (2 \beta z), \beta$ a real constant, $a=\beta^{2}$

Case III $f(z)=\cosh ^{2} \beta z, a=\beta^{2}$

Case IV $f(z)=\left(z / z_{0}\right)^{2}, z_{0}$ a real constant, $a=0$.

It should be noted that $a$ also reduces to a constant when $f=$ $=\sinh ^{2} \beta z, \cos ^{2} \beta z$, etc. and, for variations in the $x$ and $z$ directions, when $f=\exp (\beta z) \exp (\alpha x), \cosh ^{2} \beta z \cosh ^{2} \alpha x$, etc., where $\alpha$ is a real constant. In the sequel, in the interests of brevity, only cases I, II, and III will be treated.

*) This restriction is required, since the final assumption of a quasi-static response is untenable when the load is travelling into a region of changing elastic properties. 
2.1. Solutions. On making the change of variables

$$
x^{\prime}=x+v t, z^{\prime}=z, t^{\prime}=t
$$

and assuming a quasi static state, i.e. all quantities are independent of $t^{\prime}$, eqs. (2), (4), and (5) become, on deleting the primes and taking the bilateral Laplace transform with respect to $x$,

$$
\begin{gathered}
\frac{\mathrm{d}^{2} \bar{u}}{\mathrm{~d} z^{2}}-\left[\left(M^{2}-1\right) p^{2}+a\right] \bar{u}=0 \\
\bar{\sigma}_{y z}=\mu_{0} f^{\frac{1}{2}}\left(\frac{\mathrm{d} \bar{u}}{\mathrm{~d} z}-\frac{1}{2 f} \frac{\mathrm{d} f}{\mathrm{~d} z} \bar{u}\right) \\
\bar{\sigma}_{y z}=\sigma_{0}, z=0,
\end{gathered}
$$

where $M^{2}=v^{2} / c_{\mathrm{s}}^{2}, c_{\mathrm{s}}^{2}=\mu_{0} f / \rho\left(=\mu_{0} / \rho_{0}, \rho_{0}\right.$ a constant $)$ and the bar denotes the Laplace transform, parameter $p$. The solution of (7) which is bounded as $z \rightarrow \infty$ is

$$
\bar{u}=A \exp \left\{-\left[\left(M^{2}-1\right) p^{2}+a\right]^{\frac{1}{2}} z\right\},
$$

where $A$ is an arbitrary parameter and the branch of the square root is chosen such that its real part is positive when $\operatorname{Re} p>0$. Inserting (10) into (8), and then applying (9), $A$, and hence the transformed field quantities are obtained. There results for the stresses:

$$
\begin{array}{ll}
\text { Case I } & \bar{\sigma}_{y z}=\sigma_{0} \exp \left[-\left(M^{2}-1\right)^{\frac{1}{2}} p z\right] \\
\text { Case II } & \mathrm{e}^{-\beta z} \bar{\sigma}_{y z}=\sigma_{0} \exp \left\{-\left[\left(M^{2}-1\right) p^{2}+\beta^{2}\right]^{\frac{1}{2}} z\right\} \\
\text { Case III } & \frac{\bar{\sigma}_{y z}}{\sigma_{0}}=\left\{\cosh \beta z+\frac{\beta \sinh \beta z}{\left[\left(M^{2}-1\right) p^{2}+\beta^{2}\right]^{\frac{1}{2}}}\right\} \times \\
& \times \mathrm{e}^{-\left[\left(M^{2}-1\right) p^{2}+\beta^{2}\right]^{1} z} .
\end{array}
$$

2.2 Results for supersonic load speeds. For supersonic load speeds $(M>1)$, eq. (11) can be inverted by inspection and (12) and (13) can be inverted by means of the result (Magnus et al [5], p. 132)

$$
L^{-1}\left\{\frac{1}{\left(p^{2}+\gamma^{2}\right)^{\frac{1}{2}}} \mathrm{e}^{-\left(p^{2}+p^{2}\right) \xi \xi}\right\}=J_{0}\left[\gamma\left(x^{2}-\xi^{2}\right)^{\frac{1}{2}}\right] H(x-\xi),
$$

where $\mathrm{L}^{-1}$ denotes the inverse Laplace transform, $J_{0}$ denotes a Bessel function of the first kind of order zero, and $H$ denotes the 
Heaviside step function. There results:

Case I $\quad \sigma_{y z}=\sigma_{0} \delta(\eta)$

Case II $\frac{\sigma_{y z}}{\sigma_{0}}=\mathrm{e}^{\beta z} \delta(\eta)-\frac{\beta z \mathrm{e}^{\beta z}}{\zeta} J_{1}\left[\frac{\beta \zeta}{\left(M^{2}-1\right)^{\frac{1}{2}}}\right] H(\eta)$

Case III $\frac{\sigma_{y z}}{\sigma_{0}}=\cosh \beta z \delta(\eta)+\left\{\frac{\beta \sinh \beta z}{\left(M^{2}-1\right)^{\frac{1}{2}}} J_{0}\left[\frac{\beta \zeta}{\left(M^{2}-1\right)^{\frac{1}{2}}}\right]-\right.$

$$
\left.-\frac{\beta z}{\zeta} \cosh \beta z J_{1}\left[\frac{\beta \zeta}{\left(M^{2}-1\right)^{\frac{2}{2}}}\right]\right\} H(\eta)
$$

where

$$
\eta=x-\left(M^{2}-1\right)^{\frac{1}{2}} z, \zeta=\left[x^{2}-\left(M^{2}-1\right) z^{2}\right]^{\frac{1}{2}} .
$$

2.3. Results for subsonic load speeds. For subsonic load speeds $(M<1)$, branch points appear on the real axis in the $p$-plane and the details of the analysis become somewhat complicated. Solutions can be found more directly on taking a Fourier transform with respect to $x$ instead of a bilateral Laplace transform. The analysis then proceeds in a fashion similar to before and inversion is achieved by inspection for case I and for cases II and III by means of the result ([5], p. 118)

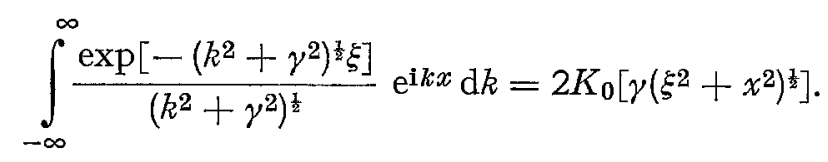

There results:

$$
\text { Case I } \quad \frac{\sigma_{y z}}{\sigma_{0}}=\frac{\left(1-M^{2}\right)^{\frac{1}{2}} z}{\pi \psi^{2}}
$$

Case II $\frac{\sigma_{y z}}{\sigma_{0}}=\frac{\beta z \mathrm{e}^{\beta z}}{\pi \psi} K_{1}\left[\frac{\beta \psi}{\left(1-M^{2}\right)^{\frac{1}{2}}}\right]$

Case III $\frac{\sigma_{y z}}{\sigma_{0}}=\frac{\beta z \cosh \beta z}{\pi \psi} K_{1}\left[\frac{\beta \psi}{\left(1-M^{2}\right)^{\frac{1}{2}}}\right]+$

$$
+\frac{\beta \sinh \beta z}{\pi\left(1-M^{2}\right)^{\frac{1}{2}}} K_{0}\left[\frac{\beta \psi}{\left(1-M^{2}\right)^{\frac{1}{2}}}\right],
$$

where

$$
\psi=\left[x^{2}+\left(1-M^{2}\right) z^{2}\right]^{\frac{1}{2}}
$$

and $K$ denotes a modified Bessel function. 
2.4. Inhomogeneity effects. The effects of inhomogeneity for supersonic load speeds are clearly shown by (14), (15), and (16). All stresses have the conical structure characteristic of supersonic speeds and all are singular - in the manner of a delta function across the conical front. The major difference between the responses is the appearance in the inhomogeneous media of an oscillatory type "wake" behind the load. The detailed structure of this wake depends on the type of inhomogeneity in question, as evidenced by (15) and (16).

For subsonic speeds all stresses again have the same type of singularity under the load, as can be seen from (17), (18), and (19), and the small-argument behavior of the modified Bessel functions. The singularity is similar in its behavior to a delta function and is of the same type as that obtained by Cole and Huth [6] in an analogous normal line load problem involving a homogeneous medium. The behavior away from the load is different in all cases, as can be seen for example on considering variations along the rays $x=b z$, where $b$ is some constant. For large values of $z$ the stresses in the homogeneous solid decay like $1 / z$, whereas the large-argument asymptotic expansions of the modified Bessel functions show that in case II, for instance, the decay is like

$$
\frac{\text { const. }}{(z)^{\frac{1}{2}}} \exp \left\{-\beta z\left[\frac{b^{2}}{\left(1-M^{2}\right)}+1\right]^{\frac{1}{3}}+\beta z\right\} \text {. }
$$

Thus the stresses in the inhomogeneous solid decay more rapidly than those in the homogeneous medium, except directly under the load $(b=0)$, where the situation is reversed. It is interesting to note that in general the greater the degree of inhomogeneity (i.e., the larger $\beta$ ), the greater is the rate of decay.

\section{§ 3. More complex physical situations}

Finally we examine more complex physical situations, wherein several types of wave motion can arise, such as the case of a moving, concentrated, normal, line load. A simple model providing a framework within which some information can be found is a solid for which

$$
\mu=\mu_{0}\left(\frac{z}{z_{0}}\right)^{2}, \quad \lambda=\lambda_{0}\left(\frac{z}{z_{0}}\right)^{2}, \quad \rho=\rho_{0}\left(\frac{z}{z_{0}}\right)^{2}, \quad \lambda_{0}=\mu_{0},
$$


where $\lambda_{0}, \rho_{0}$, and $z_{0}$ are constants. Such a medium admits of planestrain motions and - important in the solution procedure - can be described in terms of potential equations (see [3]).

For the solid defined by (20), the equations of motion are, on introducing moving coordinates, * and applying the quasi static assumption,

$$
\begin{aligned}
& \frac{\partial^{2} \phi_{1}}{\partial z^{2}}-\beta_{\mathbf{L}}^{2} \frac{\partial^{2} \phi_{1}}{\partial x^{2}}=0 \\
& \frac{\partial^{2} \phi_{2}}{\partial z^{2}}-\beta_{T}^{2} \frac{\partial^{2} \phi_{2}}{\partial x^{2}}=0,
\end{aligned}
$$

where

$$
\begin{aligned}
\beta_{\mathrm{L}}^{2} & =M_{\mathrm{L}}^{2}-1, \beta_{\mathrm{T}}^{2}=M_{\mathrm{T}}^{2}-1 \\
M_{\mathrm{L}}^{2} & =v^{2} / c_{\mathrm{d}}^{2}, M_{\mathrm{T}}^{2}=v^{2} / c_{\mathrm{s}}^{2} \\
c_{\mathrm{s}}^{2} & =\mu_{0} / \rho_{0}, c_{\mathrm{d}}^{2}=\left(\lambda_{0}+2 \mu_{0}\right) / \rho_{0} \quad\left(=3 c_{\mathrm{s}}^{2} \text { for } \lambda_{0}=\mu_{0}\right),
\end{aligned}
$$

and $v$ is the load speed (constant). The displacement-potential relations are

$$
\begin{aligned}
& z u_{x}=\frac{\partial \phi_{1}}{\partial x}+\frac{\partial^{2} \phi_{2}}{\partial x \partial z} \\
& z u_{z}=\frac{\partial \phi_{1}}{\partial z}-\frac{\partial^{2} \phi_{2}}{\partial x^{2}},
\end{aligned}
$$

and the pertinent stress-displacement relations are

$$
\begin{aligned}
\sigma_{z z} & =(\lambda+2 \mu) \frac{\partial u_{z}}{\partial z}+\lambda \frac{\partial u_{x}}{\partial x} \\
\sigma_{z x} & =\mu\left(\frac{\partial u_{z}}{\partial x}+\frac{\partial u_{x}}{\partial z}\right) .
\end{aligned}
$$

The boundary conditions are

$$
\begin{aligned}
& \sigma_{z z}=-\sigma_{0} \delta(x) \\
& \cdot \cdot \cdot \cdot \text { for } z=z_{0} . \\
& \sigma_{z x}=0
\end{aligned}
$$

For supersonic speeds $* *\left(M_{\mathbf{L}}>1\right)$ the solution procedure is

*) These are the coordinates defined by (6). The primes are deleted for convenience.

**) This is the only case treated here, since it appears that no closed form solution can be obtained for subsonic $\left(M_{T}<1\right)$ or intermediate speeds, using either transform methods or the complex variable techniques employed by Cole and Huth [6]. 
identical to that used in the SH problems. A bilateral Laplace transform with respect to $x$ is applied to (21) through (27). Bounded solutions to the ordinary differential equations arising from (21) and (22) are readily obtained and the arbitrary parameters involved can be found using the transformed versions of (23) through (27). Then all transformed field quantities can be found and the process yields for the displacements:

$$
\begin{aligned}
& \frac{\mu_{0} z \bar{u}_{x}}{\sigma_{0} z_{0}^{2}}=\frac{1}{D}\left\{\left(1+2 \beta_{\mathrm{L}} z_{0} p\right)\right. \beta_{\mathrm{T}} \mathrm{e}^{-\beta_{\mathrm{T}}\left(z-z_{0}\right) p}- \\
&\left.-\left[\beta_{\mathrm{T}}+\left(\beta_{\mathrm{T}}^{2}-1\right) z_{0} p\right] \mathrm{e}^{-\beta_{\mathbf{L}}\left(z-z_{0}\right) p}\right\} \\
& \frac{\mu_{0} z \bar{u}_{z}}{\sigma_{0} z_{0}^{2}}=\frac{1}{D}\left\{\beta_{\mathrm{L}}\left[\beta_{\mathrm{T}}+\left(\beta_{\mathrm{T}}^{2}-1\right) z_{0} p\right] \mathrm{e}^{-\beta_{\mathbf{L}}\left(z-z_{0}\right) p}+\right. \\
&\left.+\left(1+2 \beta_{\mathbf{L}} z_{0} p\right) \mathrm{e}^{-\beta_{\mathrm{T}}\left(z-z_{0}\right) p}\right\},
\end{aligned}
$$

where

$$
\begin{aligned}
& D=R\left(z_{0} p\right)^{2}+M_{\mathrm{T}}^{2}\left(3 \beta_{\mathbf{L}}+\beta_{\mathrm{T}}\right)\left(z_{0} p\right)+3\left(1+\beta_{\mathrm{L}} \beta_{\mathrm{T}}\right) \\
& R=\left(M_{\mathrm{T}}^{2}-2\right)^{2}+4 \beta_{\mathrm{L}} \beta_{\mathrm{T}} .
\end{aligned}
$$

Inversion of the Laplace transforms can be carried out by residue theory, since the only singularities which arise are the poles at the zeros of $D$ (which is related to the function determining the Rayleigh wave speed in the medium). A numerical investigation of these zeros shows them to be real and negative for all values of the load speed $v$ such that $M_{\mathrm{L}}>1$. Applying the residue theorem, eqs. (28) and (29) give

$$
\begin{gathered}
\frac{\mu_{0}}{\sigma_{0}}\left(\frac{z}{z_{0}}\right) u_{x}=\frac{\beta_{\mathrm{T}}}{\Phi}\left(w_{l} \mathrm{e}^{-\left(l / z_{0}\right) \eta_{\mathrm{T}}}-w_{m} \mathrm{e}^{-\left(m / z_{0}\right) \eta_{\mathrm{T}}}\right) H\left(\eta_{\mathrm{T}}\right)+ \\
\quad+\frac{1}{\Phi}\left(q_{m} \mathrm{e}^{-\left(m / z_{0}\right) \eta_{\mathrm{L}}}-q_{l} \mathrm{e}^{-\left(l / z_{0}\right) \eta_{\mathrm{L}}}\right) H\left(\eta_{\mathrm{L}}\right) \\
\frac{\mu_{0}}{\sigma_{0}}\left(\frac{z}{z_{0}}\right) u_{z}=\frac{\beta_{\mathrm{L}}}{\Phi}\left(q_{l} \mathrm{e}^{-\left(l / z_{0}\right) \eta_{\mathrm{L}}}-q_{m} \mathrm{e}^{-\left(m / z_{0}\right) \eta_{\mathrm{L}}}\right) H\left(\eta_{\mathrm{L}}\right)+ \\
+\frac{1}{\Phi}\left(w_{l} \mathrm{e}^{-\left(l / z_{0}\right) \eta_{\mathrm{T}}}-w_{m} \mathrm{e}^{\left.-\left(m / z_{0}\right) \eta_{\mathrm{T}}\right) H\left(\eta_{\mathrm{T}}\right),}\right.
\end{gathered}
$$

where

$$
\begin{aligned}
& \Phi=\left[M_{\mathrm{T}}^{4}\left(3 \beta_{\mathrm{L}}+\beta_{\mathrm{T}}\right)^{2}-12 R\left(1+\beta_{\mathrm{L}} \beta_{\mathrm{T}}\right)\right]^{\frac{1}{2}} \\
& 2 R l=M_{\mathrm{T}}^{2}\left(3 \beta_{\mathrm{L}}+\beta_{\mathrm{T}}\right)+\Phi \\
& 2 R m=M_{\mathrm{T}}^{2}\left(3 \beta_{\mathrm{L}}+\beta_{\mathrm{T}}\right)-\Phi
\end{aligned}
$$




$$
\begin{aligned}
\eta_{\mathbf{T}} & =x-\beta_{\mathbf{T}}\left(z-z_{0}\right), \eta_{\mathbf{L}}=x-\beta_{\mathrm{L}}\left(z-z_{0}\right) \\
w_{l} & =1-2 \beta_{\mathbf{L}} l, w_{m}=1-2 \beta_{\mathbf{L}} m \\
q_{l} & =\beta_{\mathbf{T}}-l\left(\beta_{\mathbf{T}}^{2}-1\right), q_{m}=\beta_{\mathbf{T}}-m\left(\beta_{\mathbf{T}}^{2}-1\right) .
\end{aligned}
$$

The results for the homogeneous solid (Lamé constants $\lambda_{0}, \mu_{0}$ ) can be found in an identical fashion (or the method employed in [6] can be used). They are

$$
\begin{aligned}
& \frac{\mu_{0}}{\sigma_{0}} u_{x}=\frac{2 \beta_{\mathrm{L}} \beta_{\mathbf{T}}}{R} H\left(\eta_{\mathrm{T}}\right)-\frac{\left(M_{\mathrm{T}}^{2}-2\right)}{R} H\left(\eta_{\mathrm{L}}\right) \\
& \frac{\mu_{0}}{\sigma_{0}} u_{z}=\frac{\left(M_{\mathrm{T}}^{2}-2\right) \beta_{\mathrm{L}}}{R} H\left(\eta_{\mathrm{L}}\right)+\frac{2 \beta_{\mathrm{L}}}{R} H\left(\eta_{\mathrm{T}}\right) .
\end{aligned}
$$

The solutions again display the conical structure typical of supersonic velocities. The difference between the present results and those for the SH load is that now there are two conical fronts, one associated with $\eta \mathbf{L}$ (longitudinal type wave) and one associated with $\eta_{\mathbf{T}}$ (shear type wave). The behavior under the load is also similar to the $\mathrm{SH}$ results in the sense that on letting $z=z_{0}$, $x=0+$ in (30) through (33) one finds $u_{x}$ and $u_{z}$ in the homogeneous solid to be equal to $u_{x}$ and $u_{z}$, respectively, in the inhomogeneous solid. Another feature in common between the two types of media is that in both all displacements suffer finite jumps across the conical fronts. As before, the major effect lies in the behavior behind the fronts, the displacements in the homogeneous medium being constant while those for the inhomogeneous medium decay exponentially. This exponential like wake should be contrasted with the oscillatory ones found for the SH load.* It is also interesting to note that, as can be seen from (30) and (31) and the fact that $l, m \rightarrow 0, M_{\mathrm{L}} \rightarrow \infty$, the displacements in the inhomogeneous solid go to zero as the load speed approaches infinity.

\section{§ 4. Conclusions}

In broad summary of both classes of problem, it can be concluded that the influence of inhomogeneity on the response to supersonic load speeds is not to alter the nature of any singularities, nor the

\footnotetext{
*) For the medium at hand the supersonic SH load also yields results which decay exponentially away from the front.
} 
overall structure (such as the conical fronts), but instead lies in the effects behind the fronts, the detailed structure depending on the type of inhomogeneity in question. Further work is needed to obtain the solution for the concentrated, normal, line load travelling at subsonic and intermediate speeds. These solutions would be of interest to see if effects arise in the plane-strain motions analogous to those in the SH problems, namely, the different rates of decay along different rays, the decay rates being in general greater than those occurring in homogeneous solids. Such solutions would also throw light on the interesting question of what effect, if any, does the fact that the Rayleigh waves are dispersive have on the critical load speeds.

Received 25 October 1968

Final form 26 May 1969

\section{REFERENCES}

[1] VlaAR, N. J., Bull. Seism. Soc. Am. 56 (1966) 715, 1305.

[2] Lock, M. H., Bull. Seism. Soc. Am. 53 (1963) 527.

[3] Karlsson, T. and J. F. Hook, Bull. Seism. Soc. Am. 53 (1963) 1007.

[4] Hook, J. F., J. Acoust. Soc. Am. 34 (1962) 946.

[5] Magnus, W. and F. Oberhettinger, Special Functions of Mathematical Physics, Chelsea Publ., New York 1949.

[6] Cole, J. and J. Hutr, J. Appl. Mech. 25 (1958) 433. 